\title{
Quantum-limited linewidth of a chaotic laser cavity
}

\author{
M Patra, H Schomerus, and C W J Beenakker \\ Instituut Lorentz, Universitelt Lelden, PO Box 9506, 2300 RA Leiden The Netherlands
}

(Recerved 17 May 1999, published 13 January 2000)

\begin{abstract}
A random-matrix theory is presented for the linewidth of a laser cavity in which the radiation is scattered chaotically The linewidth is enhanced above the Schawlow-Townes value by the Petermann factor $K$, due to the nonothogonality of the cavity modes The factor $K$ is expressed in terms of a non-Hermitian random matrix, and its distribution is calculated exactly for the case in which the cavity is coupled to the outside via a small opening The average of $K$ is found to depend nonanalytically on the area of the opening, and to greatly exceed the most probable value
\end{abstract}

PACS number(s) $4265 \mathrm{Sf}, 0545 \mathrm{Mt}, 4250 \mathrm{Lc}, 4260 \mathrm{Da}$

\section{INTRODUCTION}

It has been known since the conception of the laser [1] that vacuum fluctuations of the electromagnetic field ult1mately limit the narrowing of the emission spectrum by laser action This quantum-limited linewidth, or SchawlowTownes linewidth,

$$
\delta \omega=\frac{1}{2} \Gamma^{2} / I
$$

1s proportional to the square of the decay rate $\Gamma$ of the lasing cavity mode [2], and inversely proportional to the output power I (in units of photons/s) Many years later it was realized $[3,4]$ that the fundamental limit is larger than Eq (1) by a factor $K$ that characterises the nonorthogonality of the cavity modes This excess noise factor, or Petermann factor, has generated an extensive literature (see recent papers [5-9], and references therein), both because of its fundamental significance and because of its practical importance

Theories of the enhanced linewidth usually factorize $K$ $=K_{l} K$, into longitudinal and transverse factors, assuming that the cavity mode is separable into longitudinal and transverse modes Since a longitudinal or transverse mode is essentially one dimensional, that is a major simplification Separability breaks down if the cavity has an irregular shape or contains randomly placed scatteres In the language of dynamical systems, one crosses over from integrable to chaotic dynamics [10] Chaotic lasei cavities have attiacted much interest recently $[11]$, but not in connection with the quantum-limited linewidth

In this pape1 we present a general theory for the Pete1mann factor in a system with chaotic dynamics, and apply it to the simplest case of a chaotic cavity radiating through a small opening Chaotic systems require a statistical treatment, so we compute the probability distribution of $K$ in an ensemble of cavities with small variations in shape and size We find that the average of $K-1$ depends nonanalytically $\propto T \ln T^{-1}$ on the transmission probability $T$ through the opening, so that it is beyond the reach of simple perturbation theory The most probable value of $K-1$ is $\propto T$, hence it is parametrically smaller than the average

\section{RANDOM-MATRIX FORMULATION}

The spectral statistics of chaotic systems is described by random-matrix theory $[10,12]$ We begin by reformulating the existing theories for the Petermann factor $[8,9]$ in the framework of random-matrix theory Modes of a closed cavity, in the absence of absorption or amplification, are eigenvalues of a Hermitian operator $H_{0}$ For a chaotic cavity, $H_{0}$ can be modeled by an $M \times M$ Hermitian matrix with independent Gaussian-distributed elements (The limit $M \rightarrow \infty$ at fixed spacing $\Delta$ of the modes is taken at the end of the calculation ) The matrix elements are real because of timereversal symmetry (This is the Gaussian orthogonal ensemble [12]) A small opening in the cavity is described by a real, noniandom $M \times N$ coupling matı1x $W$, with $N$ the number of wave channels transmitted through the opening (For an opening of area $\mathcal{A}, N=2 \pi \mathcal{A} / \lambda^{2}$ at wavelength $\lambda$ ) Modes of the open cavity are complex ergenvalues (with a negative imaginary part) of the non-Hermitian matrix $H$ $=H_{0}-{ }_{l} \pi W W^{T}$ The scatteng matix $S$ at frequency $\omega$ is related to $H$ by $[13]$

$$
S=1-2 \pi \imath W^{T}(\omega-H)^{-1} W
$$

It is a unitary and symmetric, 1 andom $N \times N$ matrix, with poles at the eigenvalues of $H$

We now assume that the cavity is filled with a homogeneous amplifying medium (amplification rate $1 / \tau_{a}$ ) This adds a teim $l / 2 \tau_{a}$ to the eigenvalues, shiftıng them upwaids toward the real axis The lasing mode is the eigenvalue $\Omega$ $-{ }_{i} \Gamma / 2$ closest to the real axis, and the laser threshold is reached when the decay 1 ate $\Gamma$ of this mode equals the amplification rate $1 / \tau_{a}$ [14] Near the laser threshold we need to retain only the contribution from the lasing mode (say mode number $l$ ) to the scattering matrix (2),

$$
S_{n m}=-2 \pi l\left(W^{T} U\right)_{n l}\left(\omega-\Omega+{ }_{l} \Gamma / 2-\imath / 2 \tau_{a}\right)^{-1}\left(U^{-1} W\right)_{l m},
$$

where $U$ is the matrix of eigenvectors of $H$ Because $H$ is a real symmetıc matix, we can choose $U$ such that $U^{-1}$ $=U^{T}$, and wite Eq (3) in the form

$$
S_{n m}=\sigma_{n} \sigma_{m}\left(\omega-\Omega+{ }_{l} \Gamma / 2-{ }_{\imath} / 2 \tau_{a}\right)^{-1}
$$


where $\sigma_{n}=(-2 \pi l)^{1 / 2}\left(W^{T} U\right)_{n l}$ is the complex coupling constant of the lasing mode $l$ to the $n$th wave channel

The Petermann factor $K$ is given by

$$
\sqrt{K}=\frac{1}{\Gamma} \sum_{n=1}^{N}\left|\sigma_{n}\right|^{2}=\left(U^{\dagger} U\right)_{l l}
$$

The second equality follows from the definition of $\sigma_{n}$ [15], and is the matrix analogon of Siegman's nonorthogonal mode expression [4] The first equality follows from the definition of $K$ as the factor multiplying the Schawlow-Townes linewidth [16] One verifies that $K \geqslant 1$ because $\left(U^{\dagger} U\right)_{\|}$ $\geqslant\left(U^{T} U\right)_{l l}=1$

\section{SINGLE-CHANNEL CAVITY}

Relation (5) serves as the starting point for a calculation of the statistics of the Petermann factor in an ensemble of chaotic cavities Here we restrict ourselves to the case $N$ $=1$ of a single-wave channel, leaving the multichannel case for future investigation For $N=1$ the coupling matrix $W$ reduces to a vector $\vec{\alpha}=\left(W_{11}, W_{21}, \quad, W_{M 1}\right)$ Its magnitude $|\vec{\alpha}|^{2}=\left(M \Delta / \pi^{2}\right) w$, where $w \in[0,1]$ is related to the transmission probability $T$ of the single-wave channel by $T$ $=4 w(1+w)^{-2}$ We assume a basis in which $H_{0}$ is diagonal (e1genvalues $\omega_{q}$ )

If the opening is much smaller than a wavelength, then a perturbation theory in $\vec{\alpha}$ seems a natural starting point To leading order one finds

$$
K=1+\left(2 \pi \alpha_{l}\right)^{2} \sum_{q \neq l} \frac{\alpha_{q}^{2}}{\left(\omega_{l}-\omega_{q}\right)^{2}}
$$

The frequency $\Omega$ and decay rate $\Gamma$ of the lasing mode are given by $\omega_{l}$ and $2 \pi \alpha_{l}^{2}$, respectively, to leading order in $\vec{\alpha}$ We seek the average $\langle K\rangle_{\Omega \Gamma}$ of $K$ for a given value of $\Omega$ and $\Gamma[17]$ The probability to find an eigenvalue at $\omega_{q}$, given that there is an eigenvalue at $\omega_{l}$, vanishes linearly for small $\left|\omega_{q}-\omega_{l}\right|$, as a consequence of ergenvalue repulsion constrained by time-reversal symmetry Since expression (6) for $K$ diverges quadratically for small $\left|\omega_{q}-\omega_{l}\right|$, we conclude that $\langle K\rangle_{\Omega \Gamma}$ does not exist in perturbation theory This severely complicates the problem

We have succeeded in obtaining a finite answer for the average Petermann factor by starting from the exact relation

$$
U_{q l} z_{l}=\omega_{q} U_{q l}-\imath \pi \alpha_{q} \sum_{p} \alpha_{p} U_{p l}
$$

between the complex ergenvalues $z_{q}$ of $H$ and the real ergenvalues $\omega_{q}$ of $H_{0}$ Distunguishing between $q=l$ and $q \neq l$, and defining $d_{q}=U_{q l} / U_{l l}$, we obtain two recursion relations

$$
z_{l}=\omega_{l}-\imath \pi \alpha_{l}^{2}-\imath \pi \alpha_{l} \sum_{q \neq l} \alpha_{q} d_{q}
$$

$$
\iota d_{q}=\frac{\pi \alpha_{q}}{z_{l}-\omega_{q}}\left(\alpha_{l}+\sum_{p \neq l} \alpha_{p} d_{p}\right)
$$

The Petermann factor of the lasing mode $l$ follows from

$$
\sqrt{K}=\left(1+\sum_{q \neq l}\left|d_{q}\right|^{2}\right)\left|1+\sum_{q \neq l} d_{q}^{2}\right|^{-1}
$$

We now use the fact that $z_{l}$ is the ergenvalue closest to the real axis We may therefore assume that $z_{l}$ is close to the unperturbed value $\omega_{l}$, and replace the denominator $z_{l}-\omega_{q}$ in Eq (8b) by $\omega_{l}-\omega_{q}$ That decouples the two recursion relations, which may then be solved in closed forms

$$
\begin{aligned}
& z_{l}=\omega_{l}-\imath \pi \alpha_{l}^{2}(1+\imath \pi A)^{-1}, \\
& \iota d_{q}=\frac{\pi \alpha_{q} \alpha_{l}}{\omega_{l}-\omega_{q}}(1+\imath \pi A)^{-1}
\end{aligned}
$$

We have defined $A=\Sigma_{q \neq l} \alpha_{q}^{2}\left(\omega_{l}-\omega_{q}\right)^{-1}$ The decay rate of the lasing mode is

$$
\Gamma=-2 \operatorname{Im} z_{l}=2 \pi \alpha_{l}^{2}\left(1+\pi^{2} A^{2}\right)^{-1}
$$

Since the lasing mode is close to the real axis, we may linearize expression (9) for $K$ with respect to $\Gamma$,

$$
K=1+4 \sum_{q \neq l}\left(\operatorname{Im} d_{\imath}\right)^{2}=1+\frac{(2 \pi \Gamma / \Delta) B}{1+\pi^{2} A^{2}},
$$

with $B=\Delta \Sigma_{q \neq l} \alpha_{q}^{2}\left(\omega_{l}-\omega_{q}\right)^{-2}$

The conditional average of $K$ at given $\Gamma$ and $\Omega$ can be written as the ratio of two unconditional averages

$$
\begin{gathered}
\langle K\rangle_{\Omega \Gamma}=1+(2 \pi \Gamma / \Delta)\left\langle B\left(1+\pi^{2} A^{2}\right)^{-1} Z\right\rangle /\langle Z\rangle, \\
Z=\delta\left(\Omega-\omega_{l}\right) \delta\left(\Gamma-2 \pi \alpha_{l}^{2}\left(1+\pi^{2} A^{2}\right)^{-1}\right)
\end{gathered}
$$

In principle one should also require that the decay 1 ates of modes $q \neq l$ are larger than $\Gamma$, but this extra condition becomes irrelevant for $\Gamma \rightarrow 0$ For $M \rightarrow \infty$ the distribution of $\alpha_{q}$ is Gaussian $\propto \exp \left(-\frac{1}{2} \beta \alpha_{q}^{2} \pi^{2} / w \Delta\right)[12]$ with $\beta=1$ The average of $Z$ over $\alpha_{l}$ yields a factor $\left(1+\pi^{2} A^{2}\right)^{1 / 2}$,

$$
\langle K\rangle_{\Omega \Gamma}=1+(2 \pi \Gamma / \Delta) \frac{\left\langle B\left(1+\pi^{2} A^{2}\right)^{-1 / 2}\right\rangle}{\left\langle\left(1+\pi^{2} A^{2}\right)^{1 / 2}\right\rangle},
$$

where only the averages over $\alpha_{q}$ and $\omega_{q}(q \neq l)$ remain, at fixed $\omega_{l}=\Omega$

The problem is now reduced to a calculation of the joint probability distibution $P(A, B)$ This is a technical challenge, similar to the level curvature problem of randommatrix theory $[18,19]$ The calculation is given in the Appen$\mathrm{d}_{1 \mathrm{x}}$, with the result

$$
P(A, B)=\frac{1}{6} \sqrt{\frac{\pi}{2 w}} \frac{\pi^{2} A^{2}+w^{2}}{B^{7 / 2}} \exp \left[-\frac{w}{2 B}\left(\frac{\pi^{2} A^{2}}{w^{2}}+1\right)\right]
$$




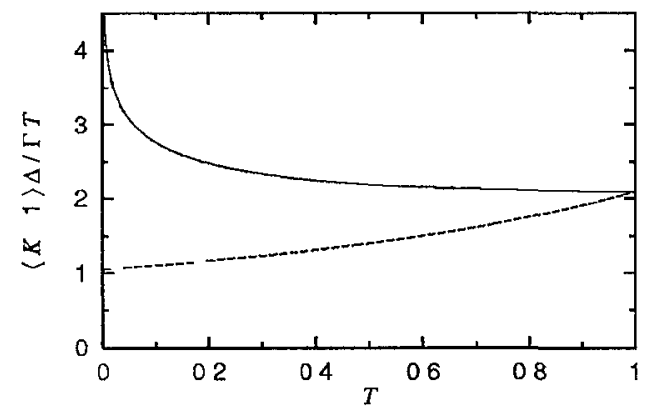

FIG 1 Avelage Petermann factor $K$ for a chaotic cavity having an opening with transmission probability $T$ The average is performed at a fixed decay rate $\Gamma$ of the lasing mode, assumed to be much smaller than the mean modal spacing $\Delta$ The solid curve is the result $[\mathrm{Eq}(16)]$ in the presence of tıme-reversal symmetry, and the dashed curve is the result [Eq (20)] for broken time reversal symmetry For small $T$, the solid curve diverges $\propto \ln T^{-1}$ while the dashed curve has the finite limit of $\pi / 3$ For $T=1$ both curves reach the value $2 \pi / 3$

Together with Eq (14), this gives the mean Petermann factor

$$
\langle K\rangle_{\Omega \Gamma}=1-\frac{\Gamma}{\Delta} \frac{2 \pi}{3} \frac{G_{22}^{22}\left(w^{2} \mid \begin{array}{cc}
0 & 0 \\
-\frac{1}{2} & -\frac{1}{2}
\end{array}\right)}{G_{22}^{22}\left(w^{2} \mid \begin{array}{ll}
-\frac{1}{2} & \frac{1}{2} \\
-1 & 0
\end{array}\right)},
$$

in terms of the ratio of two Meijer $G$ functions We have plotted the result in Fig 1 , as a function of $T=4 w(1$ $+w)^{-2}$

The non-analytic dependence of the average $K$ on $T$ (and hence on the area of the opening [20]) is a striking feature of our result For $T \ll 1$, the average 1 educes to

$$
\langle K\rangle_{\Omega \Gamma}=1+\frac{\pi}{6} \frac{T \Gamma}{\Delta} \ln \frac{16}{T}
$$

The nonanalyticity iesults from the relatively weak eigenvalue tepulsion in the presence of time-reversal symmetry If time-reversal symmetry is broken by a magneto-optical effect (as in Refs $[21,22]$ ), then the stronger quadiatic repulsion is sufficient to overcome the $\omega^{-2}$ divergence of peiturbation theory, and the average $K$ becomes an analytic function of $T$ For this case, we find, instead of $\mathrm{Eq}$ (14), the simpler expression

$$
\langle K\rangle_{\Omega \Gamma}=1+(2 \pi \Gamma / \Delta) \frac{\langle B\rangle}{\left\langle 1+\pi^{2} A^{2}\right\rangle}
$$

Using the joint probability distribution (see the Appendix)

$$
P(A, B)=\frac{\left(\pi^{2} A^{2}+w^{2}\right)^{2}}{3 w B^{5}} \exp \left[-\frac{w}{B}\left(\frac{\pi^{2} A^{2}}{w^{2}}+1\right)\right],
$$

we find the mean $K$,

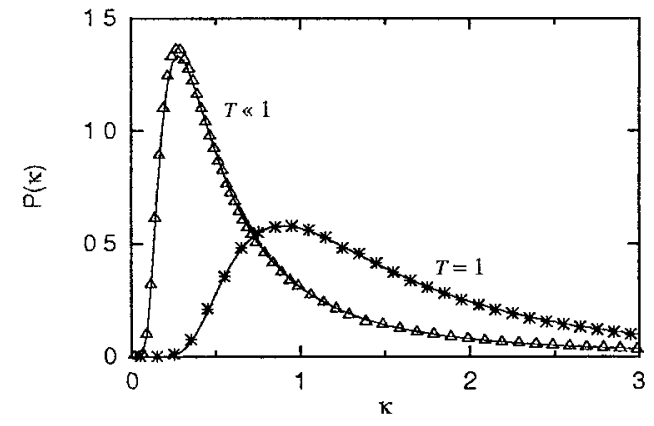

FIG 2 Probability distribution of the rescaled Petermann factor $\kappa=(K-1) \Delta / \Gamma T$ for $T=1$ and $T \ll 1$ The solid curves follow from Eqs (21) and (22) The data points follow from a numerical simulation of the random-matrix model

$$
\langle K\rangle_{\Omega \Gamma}=1+\frac{\Gamma}{\Delta} \frac{4 \pi w}{3\left(1+w^{2}\right)},
$$

shown by the dashed line in Fig 1 It is equal to $\langle K\rangle_{\Omega \Gamma}$ $=1+\frac{1}{3} \pi T \Gamma / \Delta$ for $T \ll 1$

So fal we have concentrated on the average Petermann factor, but from Eqs (11), (12), and (15) we can compute the entre probability distiobution of $K$ at fixed $\Gamma$ We define $\kappa$ $=(K-1) \Delta / \Gamma T$ A simple result for $P(\kappa)$ follows for $T=1$,

$$
P(\kappa)=\frac{4 \pi^{2}}{3} \kappa^{-7 / 2} \exp (-\pi / \kappa),
$$

and, for $T \ll 1$,

$$
P(\kappa)=\frac{\pi}{12 \kappa^{2}}\left(1+\frac{\pi}{2 \kappa}\right) \exp (-\pi / 4 \kappa), \quad \kappa T \leqq 1
$$

As shown in Fig 2, both distributions are very bioad and asymmetric, with a long tall toward large $\kappa$ [23] The most probable (or modal) value of $K-1 \simeq T \Gamma / \Delta$ is parametrically smaller than the mean value $[\mathrm{Eq}(17)]$ for $T \ll 1$

To check our analytical results, we have also done a numerical simulation of the random-matix model, generating a latge number of 1 andom matrices $H_{0}$ and computing $K$ from $\mathrm{Eq}$ (5) As one can see from Fig 2, the agreement with Eqs (21) and (22) is flawless

\section{CONCLUSIONS}

In conclusion, we have shown that chaotic scattering causes laige statistical fluctuations in the quantum-limited linewidth of a laser cavity We have examined in detall the case that the coupling to the cavity is via a single-wave chan nel, but ou random-matrix model applies more generally to coupling via an arbitrary number $N$ of wave channels $\mathrm{We}$ have computed exactly the distribution of the Petermann fac tor for $N=1$ It remains an open problem to do the same for $N>1$ This problem is related to several recent studies of the statistics of eigenfunctions of non-Hermitian Hamiltonians $[24,25]$, but is complicated by the constraint that the corre sponding eigenvalue is the closest to the real axis Our study of a system with a fully chaotic phase space complements 
previous theoretical work on systems with an integrable dynamics Chaotic laser cavities of recent experimental interest [26] have a phase space that includes both integrable and chaotic regions The study of the quantum-limited linewidth of such mixed systems is a challenging problem for future research

\section{ACKNOWLEDGMENTS}

We have benefited from discussions with $\mathrm{P} W$ Brouwer, K M Frahm, Y V Fyodorov, and F von Oppen This work was supported by the Dutch Science Foundation NWO/FOM and by the TMR program of the European Union

\section{APPENDIX: CALCULATION OF $P(A, B)$}

The joint probability distribution of the eigenvalues $w_{q}$ of $H_{0}$ is given by the Gaussian ensemble of random-matrix theory

$$
P\left(\left\{\omega_{q}\right\}\right) \propto \prod_{l<1}\left|\omega_{l}-\omega_{1}\right|^{\beta} \exp \left[-\frac{\beta \pi^{2}}{4 M} \sum_{k} \omega_{k}^{2}\right]
$$

The level spacing in the center of the semicircle has been set to unity We assume that the lasing level is at $\omega_{l}=0$ (other chorces just renormalize the level spacing) The ergenvalue distribution (A1) of the $M$-dimensional matrix $H$ then factorizes into the distribution of a $(M-1)$-dimensional matrix $H^{\prime}$ and the product $\Pi_{q \neq l}\left|\omega_{q}\right|^{\beta}=\left|\operatorname{det} H^{\prime}\right|^{\beta}$

The joint probability distribution of $A$ and $B$,

$$
P(A, B)=\left\langle\delta\left(A-\sum_{q \neq l} \frac{\alpha_{q}^{2}}{\omega_{q}}\right) \delta\left(B-\sum_{q \neq l} \frac{\alpha_{q}^{2}}{\omega_{q}^{2}}\right)\right\rangle_{\left\{\alpha_{q} \omega_{q q}\right\}}
$$

1s obtained by averaging over the variables $\alpha_{q}$ and $\omega_{q}, q$ $\neq l$ Fourier transformation of $\mathrm{Eq}$ (A2) with respect to $A$ and $B$ gives

$$
\widetilde{P}(x, y) \propto\left\langle\frac{\operatorname{det} H^{\prime 2 \beta}}{\operatorname{det}\left[H^{\prime 2}+2 l w\left(x H^{\prime}+y\right) / \pi^{2} \beta\right]^{\beta / 2}}\right\rangle_{H^{\prime}},
$$

after averaging over $\left\{\alpha_{q}\right\}$ The remaining average is over the Gaussian ensemble of $H^{\prime}$ matrices The determinant in the denominator can be expressed as a Gaussian integral,

$$
\begin{aligned}
\widetilde{P}(x, y) \propto & \int_{-\infty}^{\infty} d \mathbf{z} \int d H^{\prime} \operatorname{det} H^{\prime 2 \beta} \exp \left[-\frac{\beta \pi^{2}}{4 M} \operatorname{tr} H^{\prime 2}-\mathbf{z}^{\dagger}\right. \\
& \left.\times\left(H^{\prime 2}+\frac{2 \imath w}{\beta \pi^{2}}\left(x H^{\prime}+y\right)\right) \mathbf{z}\right]
\end{aligned}
$$

where the $M-1$ dimensional vector $\mathbf{z}$ is real (complex) for $\beta=1$ (2)

We now decompose the matrix $H^{\prime}$ as

$$
H^{\prime}=\left(\begin{array}{ll}
H^{\prime \prime} & \mathbf{h} \\
\mathbf{h}^{\dagger} & g
\end{array}\right)
$$

The $(M-2) \times(M-2)$ matrix $H^{\prime \prime}$ is distributed according to the Gaussian orthogonal ensemble, $g$ is a scalar, and the $(M-2)$-dimensional vector $\mathbf{h}$ consists of Gaussian random variables with variance

$$
h^{2} \equiv\left\langle\left|h_{\imath}\right|^{2}\right\rangle=\frac{1}{\pi^{2}} \frac{1}{B / w+1 / M} \approx \frac{w}{\pi^{2} B}\left(1-\frac{w}{M B}\right)
$$

We can always choose a basis in which $\mathbf{z}$ points in the $\mathrm{d}$ rection of the last basis vector, so that $\mathbf{z}^{\dagger} H^{\prime} \mathbf{z}=|\mathbf{z}|^{2} g$ Going back fiom Eq $(\mathrm{A} 4)$ to $P(A, B)$ by Fourier transformation, two $\delta$ functions appear, allowing to integrate over $g$ and $\mathbf{h}$ The result can be expressed as an average over $H^{\prime \prime}$ and $\mathbf{h}$

$$
\begin{gathered}
P(A, B) \propto Q_{\beta} B^{\beta / 2-2} \exp \left[-\frac{\beta w}{2 B}\left(1+\frac{\pi^{2} A^{2}}{w^{2}}\right)\right], \\
Q_{\beta}=\left\langle\operatorname{det} H^{\prime 2 \beta}\left(\frac{A}{B}-\mathbf{h}^{\dagger} V^{-1} \mathbf{h}\right)^{2 \beta}\right\rangle_{\mathbf{h} H^{\prime \prime}}
\end{gathered}
$$

For $\beta=1$ one has now to consider

$$
Q_{1}=\left\langle\operatorname{det} H^{\prime \prime 2}\left[\frac{A^{2}}{B^{2}}+h^{4}\left\{\left(\operatorname{tr} H^{\prime \prime-1}\right)^{2}+2 \operatorname{tr} H^{\prime \prime-2}\right\}\right]\right\rangle_{H^{\prime \prime}},
$$

where only the even terms in $H^{\prime \prime}$ have been kept The ratio of coefficients in this polynomial in $A / B$ can be calculated from the autocorrelator [27]

$$
\begin{aligned}
G_{1}\left(E, E^{\prime}\right) & =\frac{\left\langle\operatorname{det}\left(H^{\prime \prime}+E\right)\left(H^{\prime \prime}+E^{\prime}\right)\right\rangle}{\left\langle\operatorname{det} H^{\prime 2}\right\rangle} \\
& =-\left.\frac{3}{\pi^{2} x} \frac{d}{d x} \frac{\sin \pi x}{\pi x}\right|_{x=E-E^{\prime}}
\end{aligned}
$$

of the secular polynomial of Gaussian distributed real matrices $H^{\prime \prime}$ This is achieved by expressing the products of traces and determinants through secular coefficients, and these then as deirvatives of the secular determınant Equations (A6) and (A10) yield

$$
Q_{1} \propto \frac{A^{2}}{B^{2}}+\frac{w^{2}}{\pi^{2} B^{2}}
$$

Insertion into $\mathrm{Eq}$ (A7) and restoration of the normalization constant gives result (15)

For $\beta=2$ we have, after averaging over $\mathbf{h}$, the expression

$$
\begin{gathered}
Q_{2} \propto \frac{A^{4}}{B^{4}}+q_{1} h^{4} \frac{A^{2}}{B^{2}}+q_{2} h^{8}, \\
q_{1}=6\left\langle\operatorname{det} H^{\prime \prime 4}\left[\left(\operatorname{tr} H^{\prime \prime-1}\right)^{2}+\operatorname{tr} H^{\prime \prime-2}\right]\right\rangle_{H^{\prime \prime}},
\end{gathered}
$$




$$
\begin{aligned}
q_{2}= & \left\langle\operatorname { d e t } H ^ { \prime \prime } \left[\left(\operatorname{tr} H^{\prime \prime}\right)^{-4}+6 \operatorname{tr} H^{\prime \prime-2}\left(\operatorname{tr} H^{\prime \prime-1}\right)^{2}\right.\right. \\
& \left.\left.+8 \operatorname{tr} H^{\prime \prime-1} \operatorname{tr} H^{\prime \prime-3}+6 \operatorname{tr} H^{\prime \prime-4}+3\left(\operatorname{tr} H^{\prime \prime-2}\right)^{2}\right]\right\rangle_{H^{\prime \prime}}
\end{aligned}
$$

The coefficients can now be computed from the four-point correlator of the Gaussian unitary ensemble [28], yielding $q_{1}=2 \pi^{2}$ and $q_{2}=\pi^{2}$, thus

$$
Q_{2} \propto Q_{1}^{2}
$$

Combining the results and restoring the normalization constant, we arrive at Eq (19)
[1] A L Schawlow and C H Townes, Phys Rev 112, 1940 (1958)

[2] It is assumed that $\Gamma$ is much less than the linewldth of the atomic transition, and also that the lower level of the transition is unoccupied

[3] K Petermann, IEEE J Quantum Electron 15, 566 (1979)

[4] A E Siegman, Phys Rev A 39, 1253 (1989), 39, 1264 (1989)

[5] Y -J Cheng, C G Fanning, and A E Siegman, Phys Rev Lett 77, 627 (1996)

[6] M A van Eijkelenborg, $\AA$ M Lindberg, M S Thyssen, and J P Woerdman, Phys Rev Lett 77, 4314 (1996)

[7] M Brunel, G Ropars, A Le Floch, and F Bretenaker, Phys Rev A 55, 4563 (1997)

[8] P Giangıer and J-P Poizat, Eur Phys J D 1, 97 (1998)

[9] A E Siegman, Phys Rev A (to be publıshed)

[10] F Haake, Quantum Stgnatures of Chaos (Springeı, Berlın, 1991)

[11] J U Nockel and A D Stone, Nature (London) 385, 45 (1997)

[12] M Mehta, Random Matrices (Academic, New York, 1990)

[13] J J M Verbaarschot, H A Weidenmuller, and M R Zirnbauer, Phys Rep 129, 367 (1985)

[14] T S Misurpashaev and C W J Beenakke,, Phys Rev A 57, 2041 (1998)

[15] To prove the second equality in Eq (5), write

$$
\begin{aligned}
\Sigma_{n}\left|\sigma_{n}\right|^{2} & =2 \pi\left(U^{\dagger} W W^{T} U\right)_{l l}=2 \imath\left(U^{\dagger}\left(H-H_{0}\right) U\right)_{l l} \\
& =2 \imath\left(U^{\dagger} U\right)_{l l}\left(\omega_{0}-{ }_{\imath} \Gamma / 2\right)-2{ }_{l}\left(U^{\dagger} H_{0} U\right)_{l l},
\end{aligned}
$$

and take the real part

[16] Fol the first equality in Eq (5), wite the linewidth $\delta \omega=\Gamma$ $-1 / \tau_{a}$ in terms of the output power $I=\int \mathrm{t} 1 S S^{\dagger} d \omega / 2 \pi$ $=\left(\Sigma_{n}\left|\sigma_{n}\right|^{2}\right)^{2}\left(\Gamma-1 / \tau_{a}\right)^{-1}=K \Gamma^{2} / \delta \omega$ The linewidth diffe1s from the Schawlow-Townes value $[\mathrm{Eq}(1)]$ by a factor $2 K$
The extra factor 2 arises from the suppression of amplitude fluctuations in the nonlinear legime above the laser threshold, as explamed by P Goldberg, P W Milonni, and B Sundaram, Phys Rev A 44, 1969 (1991)

[17] From the conditional average $\langle K\rangle_{\Omega} \Gamma$ of the Petermann factor at given $\Omega$ and $\Gamma$, one may compute the unconditional average $\langle K\rangle=\int d \Omega \int d \Gamma P(\Omega, \Gamma)\langle K\rangle_{\Omega \Gamma}$ The distribution $P(\Omega, \Gamma)$ of the lasing mode was calculated in Ref [14]

[18] F von Oppen, Phys Rev Lett 73, 798 (1994), Phys Rev E 51, 2647 (1995)

[19] Y V Fyodolov and H-J Sommers, Z Phys B 99, 123 (1995)

[20] The transmission probability $T$ is related to the area $\mathcal{A}$ of the opening by $T \simeq \mathcal{A}^{3} / \lambda^{6}$ for $T \ll 1$ See $\mathrm{H}$ A Bethe, Phys Rev 66, 163 (1944)

[21] H Alt, H -D Graf, H L Harney, R Hofferbert, H Lengeler, A Richter, P Schardt, and H A Weidenmuller, Phys Rev Lett 74, 62 (1995)

[22] U Stoffregen, J Steın, H -J Stockmann, M Kuś, and F Haake, Phys Rev Lett 74, 2666 (1995)

[23] For the case of broken time-reversal symmetry, we find $P(\kappa)=\frac{8}{3} \pi^{4} \kappa^{-5} \exp (-2 \pi / \kappa) \quad$ for $\quad T=1, \quad$ and $P(\kappa)$ $=\frac{1}{6} \pi 2^{-1 / 2} \kappa^{-5 / 2}\left[\frac{3}{4}+\pi / 2 \kappa+(\pi / 2 \kappa)^{2}\right] \exp (-\pi / 2 \kappa)$ for $T \ll 1$

[24] J T Chalker and B Mehlig, Phys Rev Lett 81, 3367 (1998)

[25] R A Jank, W Norenberg, M A Nowak, G Papp, and I Zahed, Phys Rev E 60, 2699 (1999)

[26] C Gmachl, F Capasso, E E Narumanov, J U Nockel, A D Stone, J Farst, D L Sivco, and A Y Cho, Science 280, 1556 (1998)

[27] S Kettemann, D Klakow, and U Smlansky, J Phys A 30, 3643 (1997)

[28] A V Andreev and B D Simons, Phys Rev Lett 75, 2304 (1995) 